\title{
Las compras públicas de tecnología e innovación como un elemento central de las políticas públicas del siglo XXI
}

\author{
Public Procurement of Technology and Innovation \\ as a Key Element in Public Policies in the $21^{\text {sT }}$ Century
}

CÉSAR ABUSLEME MARDONES

Abogado, Ministerio de Hacienda, Chile

RESUMEN Este artículo tiene por objeto describir los principales problemas y oportunidades que existen para que el Estado pueda adquirir nuevas tecnologías y soluciones innovadoras. En este sentido, se analizan conceptos básicos en la materia como innovación pública y compra pública de innovación, así como el estado actual del gobierno digital en Chile. Además, se hace una revisión crítica de las iniciativas públicas realizadas en Chile y de los enfoques académicos en la materia. Finalmente, se estudian procedimientos especiales utilizados en el extranjero para la adquisición de innovación.

PALABRAS CLAVE Innovación pública, gobierno digital, compras públicas.

ABSTRACT The following essay describes the main problems and opportunities regarding the procurement of new technologies and innovative solutions by the government. Accordingly, basic concepts, such as public innovation, and public procurement of innovation, are analyzed, as well as the digital government's current situation in Chile. Moreover, both 
Chilean public measures and academic approaches on this matter are critically examined. Finally, special procedures used for procuring innovation are also studied.

KEYWORDS Public innovation, digital government, public procurement.

\section{INTRODUCCIÓN}

La innovación pública es, desde hace muchos años, un tema muy en boga en la literatura sobre gestión pública, nacional e internacional. Sin embargo, la doctrina jurídica chilena aún no ha mostrado interés en la materia, permitiendo la existencia de una brecha académica entre la literatura legal y la literatura sobre políticas públicas. Así, por ejemplo, desde esta última trinchera, se ha relativizado el rol de la ley como un límite de la innovación pública, ${ }^{\mathrm{I}}$ postulando que los privados también deben enfrentar restricciones legales y que, por ende, el derecho no merece una consideración especial en este sentido (Sánchez y Lasagna, 20I3: 32). Sin embargo, la doctrina administrativa nacional considera que la Administración, además de someterse al imperio del derecho, como hacen también los sujetos privados, sólo puede, en virtud del principio de juridicidad, realizar aquello que expresamente le ha sido autorizado por el ordenamiento jurídico (Bermúdez, 2015: 275). Ambas posturas son claramente contradictorias, lo que hace manifiesta la necesidad de acercamiento entre estas dos escuelas.

Sin perjuicio de lo anterior, el estudio del derecho y la tecnología se ha transformado en una de las áreas donde existe mayor conexión entre la doctrina jurídica y los asuntos públicos. En la actualidad, el tratamiento de datos personales en el sector público, la protección de la vida privada en el uso de medios digitales y la publicación de datos abiertos por parte de los organismos gubernamentales, entre otros temas, han sido debidamente tratados desde la perspectiva jurídica. Esto, por supuesto, se debe

I. En el ejemplo, la ley es concebida como una restricción de la innovación. Sin embargo, la ley, como es evidente, también puede constituirse en una herramienta clave para favorecer la innovación o, incluso, en el objeto de la misma, como sucede, por ejemplo, en los grandes procesos de reforma de los sistemas judiciales. 
a que los avances tecnológicos han cambiado la forma en que la ciudadanía interactúa con el Estado en la era digital. Con todo, existen temas sobre los cuales la doctrina jurídica chilena no ha emitido prácticamente pronunciamiento alguno, como es el caso de la innovación pública y, más en específico, de las compras públicas de tecnología e innovación.

En el Chile del siglo XXI, las personas pueden realizar toda clase de trámites a través de medios digitales, desde aquellos más simples, como la emisión de certificados de nacimiento, defunción y dominio, hasta otros mucho más complejos, como el cumplimiento de las obligaciones tributarias o, incluso, la tramitación de causas judiciales. En este contexto, resulta evidente que el Estado es y seguirá siendo en el futuro un gran consumidor de tecnología. De ahí la importancia de estudiar normativamente el estado de las compras públicas de tecnología e innovación.

En el presente trabajo, se tratará de hacer una primera bajada conceptual al tratamiento de este asunto. Así, se pretende evitar que cuestiones como el gobierno digital y la innovación se transformen en conceptos vacíos. Para ello, el presente trabajo se divide en tres secciones principales. En una primera sección, se describe el contexto tecnológico en el cual las compras públicas se encuentran insertas en la actualidad, poniendo especial énfasis en los requerimientos de un Estado moderno y en las bases de un futuro gobierno digital. En una segunda sección, se le entrega un contenido concreto a los conceptos de innovación e innovación pública, para luego revisar en específico la conexión entre la regulación de compras públicas y la adquisición de nuevas tecnologías. Finalmente, en una tercera sección, se tratan ciertos procedimientos de compras públicas especiales que tienen directa relación con la adquisición de tecnologías innovadoras.

El objetivo, entonces, es delinear con certeza los conceptos que gobiernan la compra pública de innovación y tecnología en la actualidad. Se espera que este trabajo sirva para incentivar en Chile el debate académico sobre otros temas directamente relacionados y que han sido largamente discutidos en la doctrina extranjera, como aquellos referidos a los instrumentos más adecuados para realizar compras públicas de innovación (Georghiou y otros, 20I4), la utilización de los procedimientos de compras públicas para promover la innovación en el sector privado (Aschhoff y Sofka, 2008; Edquist y Zabala-Iturriagagoitia, 20 I 2), la medición del impacto de las compras públicas en la innovación (Appelt 
y Galindo-Rueda, 2016) y las ventajas de las alianzas público-privadas por sobre los procedimientos de compras públicas más tradicionales (Hoppe y otros, 20I3).

\section{EL ROL DE LAS COMPRAS PÚBLICAS EN LA ERA DIGITAL}

\section{LAS POLÍTICAS PÚBLICAS EN LA ERA DIGITAL}

De acuerdo a la Organización para la Cooperación y Desarrollo Económico (OCDE), Chile enfrenta diversas presiones presupuestarias, por lo que el Estado debiera hacer un mejor uso de las tecnologías en el diseño e implementación de políticas públicas, para que éstas sean capaces de responder a las exigencias de la ciudadanía (OCDE, 20I6: I3). A este respecto, debe tenerse presente que la utilización de tecnologías de la información y comunicaciones (TIC) en el sector público supone ciertas dificultades que lo distinguen del sector privado (Reddick, 20I 2: 7-8). En primer lugar, los organismos públicos suelen tener objetivos más complejos y ambiguos que las empresas privadas. En segundo lugar, las instituciones públicas están sujetas a una serie de reglas burocráticas mucho más estrictas. Y, en tercer lugar, tienen procedimientos mucho más formalizados para la contratación de personal, la adquisición de bienes y servicios y muchas otras tareas administrativas.

Así, entonces, existe una contradicción fundamental entre los requerimientos de un Estado moderno y el diseño estructural del Estado actual. Por una parte, se reconoce la importancia de la innovación y de las nuevas tecnologías para hacer frente a las demandas ciudadanas, y, por otra parte, existe un diseño gubernamental conservador, burocrático y en exceso formalizado. Sin duda, una de las áreas donde esta contradicción se hace más evidente es en la regulación de las compras públicas, como se mostrará más adelante en el presente trabajo.

En este contexto, se ha hecho necesario que los Estados tengan una política clara y bien definida en materia de gobierno digital, esto es, respecto del uso de tecnologías digitales como parte de sus estrategias de modernización para crear valor público (OCDE, 20I4: 6). Los gobiernos han tendido a enfocar sus políticas públicas en esta materia en tres áreas principales: la reforma o modernización de los órganos públicos, la adecuación de la infraestructura pública para facilitar el uso de sistemas di- 
gitales, y el impacto social y económico que implica el uso de tecnologías en el Estado (McIver y Elmagarmid, 2008: 23). Con todo, siempre debe tenerse presente que la utilización de las TIC tiene impactos diferentes según el área de que se trate (tributación, escrutinio, compras públicas, etcétera) y que, asimismo, el referido impacto está condicionado por la calidad de las instituciones, la infraestructura, la tecnología y el capital humano de cada país o sector (Kochanova y otros, 2016: 3).

En Chile existen determinadas iniciativas que se espera sean útiles para formar las bases de un futuro gobierno digital, como son el lanzamiento de la Agenda Digital 2020 y la creación del Comité de Ministros para el Desarrollo Digital.

La Agenda Digital 2020 contiene una serie de medidas que llevará adelante el sector público para que «el uso masivo de las tecnologías se transforme en un medio para ayudar a reducir las desigualdades; para abrir más y mejores oportunidades de desarrollo y, así, contribuir al respeto de los derechos de todos los chilenos y chilenas». ${ }^{2}$ Esta Agenda tiene un eje especialmente dedicado al gobierno digital, en el cual se contemplan medidas directamente relacionadas con el apoyo a políticas sectoriales del Estado, mediante el uso de tecnologías y la promoción de un Estado más dinámico e innovador. En esta línea, la Agenda supone dar continuidad al trabajo que actualmente desarrolla el Laboratorio de Gobierno, fortalecer el marco institucional del gobierno digital y mejorar las capacidades para realizar compras públicas y contrataciones TIC, cuestión que se revisará en el siguiente apartado.

El Laboratorio de Gobierno ${ }^{3}$ es una iniciativa gubernamental compuesta por un equipo multidisciplinario, encabezado por un directorio interministerial, que busca desarrollar, facilitar y promover procesos de innovación centrados en los usuarios en las instituciones públicas del Estado chileno. En este sentido, esta iniciativa cuenta con tres líneas de acción: la gestión de espacios para que los privados puedan desarrollar soluciones innovadoras; la capacitación de funcionarios públicos; y, lo que más interesa para efectos de este artículo, el apoyo a instituciones

2. Toda la información citada en el presente trabajo en referencia a la Agenda Digital 2020 se ha obtenido de la revisión del sitio web www.agendadigital.gob.cl.

3. Toda la información citada en el presente trabajo en referencia al Laboratorio de Gobierndo se ha obtenido de la revisión del sitio web www.lab.gob.cl. 
públicas en el desarrollo de proyectos de innovación. Como se verá más adelante, potenciar esta última línea de acción puede ser fundamental para modificar ciertos paradigmas en relación con la eficiencia, que impiden la explotación al máximo de la capacidad innovadora del Estado.

Por otra parte, en estrecha relación con el lanzamiento de la Agenda Digital 2020, el gobierno, mediante el Decreto I de 2016, del Ministerio Secretaría General de la Presidencia, creó el Comité de Ministros para el Desarrollo Digital, instancia que tiene por objeto «proponer las políticas públicas, planes, programas y acciones específicas destinadas a generar valor económico, político y social mediante el uso de las tecnologías de información y comunicación». Si bien su misión parece estar alineada con los máximos estándares internacionales, este Comité sólo tiene la calidad de Comisión Asesora Presidencial y, por ende, sus acuerdos no son vinculantes. Como se ve, aún queda mucho camino por recorrer para que exista en Chile un gobierno digital efectivo, con una autoridad empoderada para liderar y coordinar las políticas públicas en esta materia.

En este sentido, la OCDE ha recomendado a Chile crear una nueva subsecretaría o servicio público encargado del gobierno digital, un Chief Information Officer (CIO) y, entre otros cargos, un Chief Technology Officer que responda al CIO o a quien dirija la nueva subsecretaría o servicio público de que se trate (OCDE, 2016: 82-83). El Chief Technology Officer estaría encargado, entre otras cosas, de diseñar estrategias de compras conjuntas de TIC, gestionar fondos especialmente destinados a ellas y fomentar el uso de equipos compartidos dentro de la Administración del Estado. Además, en seguimiento de estas sugerencias, se deberían establecer marcos jurídicos especiales para el desarrollo de servicios compartidos y asegurar la realización de compras conjuntas de TIC en el Estado.

En términos más concretos, el avance en materia digital supone, ente otras cosas, que Chile reemplace los procedimientos administrativos en soporte papel por la tramitación electrónica de documentos (Rivero y Fernández, 2016: 455), implemente mejores soluciones en materia de transparencia (open government) y cree nuevos sistemas de gestión de la información. Todas estas actividades requieren, como es evidente, la adquisición de tecnologías por parte del Estado y, asimismo, el éxito de éstas depende en gran medida de la calidad de los bienes y servicios contratados. 
En materia de compras públicas, la OCDE ha recomendado a sus Estados miembros adquirir tecnologías digitales en base a la evaluación de sus capacidades existentes, incluyendo competencias digitales, perfiles laborales, tecnologías, contratos y acuerdos entre diversos organismos públicos para incrementar la eficiencia, apoyar la innovación y lograr los objetivos generales de la agenda de modernización del sector público (OCDE, 20I4: 8). Además, ha insistido en que las reglas de compras y contrataciones públicas deben ser actualizadas para hacerlas compatibles con las formas más modernas de desarrollo e implementación de tecnologías digitales. Para el caso específico de Chile, la OCDE ha recomendado el desarrollo de un enfoque estratégico en materia de compras públicas de tecnologías digitales (OCDE, 2016: 44).

En esta línea, el gobierno se ha comprometido, en el marco de la Agenda Digital 2020, a actualizar el marco jurídico nacional para favorecer el desarrollo digital, mediante la estandarización de las bases de las licitaciones públicas para comprar tecnología y la revisión de la normativa asociada a estos procesos de compra, con la intención de permitir una mayor interacción con los potenciales proveedores en la búsqueda de soluciones disponibles para resolver las necesidades públicas, lo que implica licitar desde la problemática y no desde la solución (medida núm. 5). Además, se ha obligado a promover un Estado más innovador, mediante la mejora de las competencias de los órganos compradores y de las autoridades para la contratación pública de tecnología y la elaboración de nuevas normativas, directrices y elementos que favorezcan la contratación de soluciones innovadoras (medida núm. 38).

Sin perjuicio de lo anterior, entre los hitos de cumplimiento de cada una de estas medidas, no se encuentra ningún compromiso relacionado con la modificación de la Ley I9.886 sobre Contratos Administrativos de Suministro y Prestación de Servicios (Ley de Compras) ni el Decreto 250 de 2005, del Ministerio de Hacienda, mediante el cual se aprobó el reglamento de esta ley (Reglamento de Compras Públicas). Por el contrario, sólo se establecen acciones más modestas, como la actualización de la Directiva 8 de la Dirección de Compras y Contratación Pública (ChileCompra) sobre compras tecnológicas y la construcción de una directiva sobre datos abiertos y compras públicas. La primera de estas me- 
didas se cumplió con la dictación de la Directiva 24 sobre Instrucciones para la Contratación de Bienes y Servicios relacionados con Tecnologías de Información. Esta Directiva recomienda que las contrataciones más complejas de tecnología estén asociadas a una estrategia previa, validada por las jefaturas superiores de cada servicio público y, además, que en éstas se considere la estrategia tanto gubernamental como sectorial en materia de gobierno electrónico, ${ }^{4}$ de conformidad con la alineación estratégica entre las iniciativas en materia digital y las políticas generales del gobierno sugerida por la OCDE.

\section{INNOVACIÓN Y COMPRAS PÚBLICAS}

\section{EL CONCEPTO DE INNOVACIÓN}

La revisión exhaustiva de la literatura sobre innovación en el sector público (De Vries y otros, 20 I 5: I 52) ha demostrado que la mayoría de los estudios sobre la materia no contienen una definición del concepto de innovación. Además, se ha evidenciado que la doctrina que sí provee de una conceptualización de innovación la define como una idea, práctica u objeto que es considerado nuevo por el individuo o unidad que la adopta. Por su parte, la doctrina clásica sobre innovación la entiende como la introducción de un nuevo bien, un nuevo método de producción, la apertura de un nuevo mercado o la utilización de una nueva fuente proveedora de materias primas o de nuevas formas de organización industrial (Rolfstam, 201 2: 3).

El problema con estas definiciones es que no permiten distinguir cla-

4. El concepto de gobierno electrónico o e-government ha ido evolucionando hacia el concepto de gobierno digital tratado en este trabajo. Existen diversas definiciones al respecto. Hace más de una década era entendido como «la habilidad de los gobiernos para proveer servicios en forma electrónica, aumentando la eficiencia de la gestión pública y mejorando los servicios a los ciudadanos en un marco más transparente al actual, y no simplemente por procedimientos electrónicos tradicionales» (Rodal, 2004: 339). Hoy, en términos mucho más sencillos, con una mirada retrospectiva, se ha definido como el uso por parte de los gobiernos de tecnologías de información y comunicación y, particularmente, de internet, como una herramienta para mejorar su desempeño (OCDE, 20I6: 6). Existiendo diversas nociones de ambos conceptos, las cuales muchas veces se confunden, en el presente trabajo se utilizan ambas sin distinción. 
ramente entre innovación y creatividad (o invención). En consideración de esta situación, se ha postulado que tendrían la calidad de innovadoras todas las nuevas creaciones de importancia social o económica, implementadas principalmente por empresas (Edquist y Zabala-Iturriagagoitia, 20I2: I756). Esta definición no sólo permite distinguir entre innovación e invención, sino que además le otorga un sentido práctico, agregando contenido en términos de impacto. Con todo, el rol preponderante del sector privado sobre el sector público en materia de innovación ha sido puesto en duda fuertemente durante los últimos daños y no es una cuestión pacífica entre los autores (Hartley y otros, 20I3: 822).

Siguiendo esta misma línea, existen autores que proponen que la innovación sea entendida como nuevas ideas que funcionan (Mulgan, 2003: 3) y la innovación exitosa como la creación e implementación de nuevos procesos, productos, servicios y métodos de prestación de los mismos, que redundan en mejoras importantes en términos de eficiencia, efectividad o calidad. Nótese que, además del contenido práctico que se encuentra explícito en esta definición, ésta le otorga un carácter positivo a la innovación, indicando que necesariamente funciona. Debe tenerse presente que esto no es unánime en la doctrina y que para muchos la innovación puede fracasar, sin perder su calidad de tal (Potts, 2009: 36).

Yendo un poco más lejos, se ha propuesto, además, definir innovación como un proceso complejo y repetitivo a través del cual se definen problemas; se desarrollan y mezclan nuevas ideas, se prueban, diseñan y rediseñan prototipos y pilotos; y se implementan, difunden y problematizan nuevas soluciones (Bason y otros, 20I3: I I). Esta definición pone énfasis en un punto fundamental de la innovación, en la medida en que no sólo se trata de una simple idea que se trata de implementar, sino de un proceso complejo, desarrollado en diversas etapas. La concepción de la innovación como un proceso, como se verá más adelante, permite entender mejor el rol que cumple en esta materia la regulación sobre compras públicas respecto de la adquisición de nuevas tecnologías.

En síntesis, en la literatura existe cierto consenso en cuanto a que la innovación tiene tres características esenciales, que permiten diferenciarla de otros conceptos que se encuentran en la frontera, como creatividad, cambios progresivos, emprendimiento, mejoras, etcétera. En primer lugar, la innovación implica necesariamente una idea nueva (novedad), como elemento estructural básico. En segundo lugar, esta idea debe ser 
desarrollada e implementada (implementación práctica). Finalmente, la referida idea debe ser útil o funcional, a lo menos teóricamente (utilidad), sin perjuicio de un eventual fracaso durante su etapa de implementación.

\section{INNOVACIÓN PÚBLICA}

La innovación pública se ha entendido como el proceso de generación de nuevas ideas y la implementación de las mismas para crear valor para la sociedad (Bason y otros, 20I3: I I). En esta línea, también se ha sostenido que la innovación pública comprende ideas nuevas que son útiles para la generación de valor público (Mulgan, 2007: 6). Como se puede apreciar, ambas definiciones comparten las características de novedad, implementación y utilidad anotadas más arriba. Además, estas definiciones le otorgan un sentido específico a esta clase de innovación: la generación de valor público. Los contornos de este concepto son difusos y existen diversos intentos por otorgarle un contenido específico (Kattel y otros, 20I2: I 5 ).

Sin perjuicio de lo anterior, valor público se ha definido tradicionalmente como el valor creado por el gobierno, a través de la prestación de servicios, la elaboración de leyes y otras acciones (Kelly y otros, 2002: 4). Esta definición puede parecer insuficiente, por lo que sus autores se han apresurado a dotarla de un contenido más concreto. Para ello, se ha construido un consenso respecto a que los ciudadanos son los mejores jueces para determinar si una acción del gobierno genera o no valor (Benington y Moore, 20I I: 8; Kelly y otros, 2002: 4). En este sentido, se ha postulado que el valor público puede ser medido por la ciudadanía atendiendo a la calidad de los servicios prestados, de manera similar a la que se produce en las relaciones de consumo en el sector privado; en términos de resultados (o outcomes), como son, por ejemplo, la reducción de los índices de delincuencia o de las tasas de desempleo; y, además, en términos de confianza, entendiendo ésta como la posibilidad de vivir bajo un sistema gubernamental abierto, justo y democrático (Kelly y otros, 2002: I I y ss.).

Por otra parte, se debe tener en cuenta que bajo el concepto de innovación pública caben dos clases de innovación: la innovación en o dentro del sector público y la innovación por medio del sector público (Kattel y otros, 2012: 3; Bason y otros, 20I3: II). La innovación dentro del sec- 
tor público pretende, desde un enfoque interno, mejorar la eficiencia del sector público ${ }^{5} \mathrm{y}$, desde una perspectiva externa, mejorar los servicios que el Estado entrega a los ciudadanos y a las empresas; ${ }^{6}$ mientras que la innovación por medio del sector público tiene por objeto promover la innovación en el sector privado. 7 Si bien se ha avanzado mucho en los últimos años, especialmente a través de iniciativas de la Corporación de Fomento y Producción (Corfo) en la promoción y apoyo de la innovación en el sector privado, aún queda mucho espacio para que el Estado se transforme en un actor clave del sistema nacional de innovación.

Esta distinción entre innovación pública en y por medio del sector público se hace difusa cuando se trata de impulsar la innovación utilizando los mecanismos de adquisición de bienes y servicios por parte del Estado, en la medida en que una determinada compra pública puede, al mismo tiempo, tener impacto tanto en el sector público como en el sector privado.

\section{COMPRA PÚBLICA DE INNOVACIÓN (CPI)}

Las compras públicas, en general, implican la adquisición por parte de un órgano o servicio público de determinados bienes y/o servicios. Teniendo esto presente, se ha construido una clase especial de adquisiciones, distinta a aquellas que se realizan ordinariamente (compra de offthe-shelf products), denominada Compra Pública de Innovación (CPI).

5. Ejemplo de esta clase de innovación sería, por ejemplo, la implementación de un nuevo Sistema Nacional de Gestión de la Información para el Estado. Véase Licitación Pública ID 85 I 5 56-2-LEı 6 en el portal de Mercado Público (www.mercadopublico.cl).

6. En Chile, un buen ejemplo de esta clase de innovación es el portal Crea tu Empresa en un Día (www.empresaenundia.cl) del Ministerio de Economía, Fomento y Turismo. Este portal permite a personas naturales y jurídicas crear o modificar sociedades comerciales en línea.

7. Un ejemplo concreto de innovación por medio del sector público se encuentra en el Instrumento de Financiamiento denominado "Contratos Tecnológicos para la Innovación» de la Corporación de Fomento y Producción (Corfo). Esta herramienta consiste en un concurso que tiene por objeto cofinanciar contratos entre empresas y proveedores de conocimiento, los que estas empresas, en principio, no estarían dispuestas a suscribir si parte de los costos del contrato no fueran asumidos por Corfo. Para más información dirigirse a http://www.corfo.cl/programas-y-concursos/programas/ contratos-tecnologicos-para-la-innovacion. 
En este sentido, se ha sostenido que una determinada adquisición toma la forma de una CPI cuando el Estado pretende adquirir un bien y/o un servicio indeterminado, pero exigiendo que éste cumpla con ciertas funcionalidades, dentro de un periodo de tiempo razonable, con el objeto de satisfacer ciertas necesidades (Edquist y Zabala-Iturriagagoitia, 20 I 2: I758).

Así, entonces, cuando el sector público decide comprar innovación, sólo presentaría un problema a los potenciales proveedores, quienes pueden ofrecer cualquier producto o servicio que permita resolver la problemática presentada. Esta concepción de la compra pública de innovación se encuentra implícita en la medida 5 de la Agenda Digital 2020 a la que se hizo referencia antes. En cambio, la compra tradicional de bienes y servicios supondría que el Estado ya determinó cómo debe satisfacerse una determinada necesidad y, por ende, solicita directamente una solución. ${ }^{8}$

Esta distinción, si bien parece razonable, puede llevar a equívocos indeseados (Rolfstman, 20I2: 6). Se trata de una aproximación sesgada, que invita a pensar que cuando se habla de CPI se está hablando de un proceso de compras en particular, esto es, por ejemplo, de una licitación pública específica. Sin embargo, bien puede suceder que dos o más procesos de adquisiciones formen parte de una estrategia más general en favor de la innovación. De acuerdo con lo recién planteado, la referida diferenciación desconoce, además, la posibilidad de que una compra, en apariencia común y corriente, forme parte de una estrategia de CPI más amplia. ${ }^{9}$

8. Por ejemplo, para facilitar el acceso de personas con discapacidad a las instalaciones de un determinado servicio público, éste podría realizar un llamado a licitación pública para la adquisición de rampas (compra pública tradicional) o podría simplemente indicar que se requiere un producto con ciertas funcionalidades, como no obstruir las entradas principales, tener bajos costos de manutención y ser de fácil utilización (compra pública de innovación). En el segundo caso, los oferentes podrían proponer la construcción no sólo de rampas, sino también de otros accesos especiales, pasarelas o puertas laterales, o la instalación de ascensores, entre otras posibilidades.

9. Así, por ejemplo, durante el desarrollo e implementación de los sistemas de información de los Servicios Locales de Educación propuestos en el Proyecto de Ley que crea el nuevo Sistema de Educación Pública (Boletín I0368-04), se podrían realizar procesos de contratación tanto para el diseño de los referidos sistemas como para la adquisición de 
Así, la CPI puede suponer uno o más procesos de compras, e incluso puede admitir, dentro de una estrategia determinada, la inclusión de adquisiciones más tradicionales. En este sentido, conviene tener presente la concepción, referida antes, de la innovación como un proceso. En su calidad de tal, la innovación consta de diversas etapas, siendo las más comunes, a lo menos en el plano teórico, las de investigación, desarrollo, implementación y evaluación, y cada una de ellas puede implicar diversas contrataciones, algunas de innovación propiamente tal y otras sólo en cuanto forman parte de un proyecto o estrategia cuyo fin último es la innovación.

En este contexto es preferible entender la CPI en términos mucho más sencillos, simplemente como el conjunto de actividades de compra ejercidas por agentes públicos dirigidas a producir innovación (Rolfstam, 20I2: 5). La ventaja de esta definición es que permite identificar a la CPI tanto respecto de una compra en particular, como respecto de una estrategia más amplia, lo que parece más adecuado desde la perspectiva de un futuro gobierno digital.

Con todo, la CPI también se ha definido como «una actuación administrativa de fomento de la innovación, orientada a potenciar el desarrollo de soluciones innovadoras desde el lado de la demanda, a través del instrumento de la contratación pública» (Bernal y otros, 20 I 5: 8). Con esto se pretende recalcar que la búsqueda de soluciones innovadoras no sólo tiene impacto en los servicios que entrega el Estado, sino que también puede tener una función instrumental para fomentar la innovación en el sector privado. Sin embargo, la mayoría de las veces la CPI se realiza en base a necesidades propias del sector público o de determinados desafíos sociales y no con la intención específica de fomentar la innovación privada, aunque así suceda en la práctica (Lember y otros, 20I4: I5-I6).

los bienes necesarios para su instalación (por ejemplo, software u otros insumos computacionales). Analizada aisladamente, la compra de bienes puede parecer una adquisición ordinaria. Sin embargo, se trata de una compra funcional a una estrategia de CPI más general (el desarrollo e implementación de un nuevo sistema de información). 
En general, cuando se trata de identificar la CPI con un proceso de compras en particular, este concepto se confunde con la definición de Compra Pública de Tecnología Innovadora (CPTI), otro concepto clave y, tal vez, mucho más concreto o, a lo menos, de contornos más claros. En términos sencillos, la CPTI corresponde a un proceso de compras que busca diseñar una nueva tecnología que el sector público requiere para satisfacer una necesidad determinada (Rolfstam, 20I 2: 4). En otros términos, se ha definido como «la compra pública de un bien o servicio que no existe en el momento de la compra pero que puede desarrollarse en un período de tiempo razonable» (Bernal y otros, 20 I 5: 38). Así, entonces, esta concepción de CPTI se confunde con la noción errónea de CPI que se revisó al comienzo del apartado anterior. En este sentido, lo único relevante para efectos del presente artículo es entender que al hablar de compras públicas de innovación, la CPI hace referencia a su dimensión estratégica, mientras que la CPTI dice relación con un proceso de compras en particular.

A comienzos de siglo, la literatura europea comenzó a alertar sobre la absoluta ausencia de la CPTI en el diseño de políticas públicas y, en general, en cualquier debate al respecto (Edquist y Hommen, 2000: 7). En esa época, sólo existía preocupación por regular las formas de compra más tradicionales, es decir, aquellas que no daban lugar a innovación. Una década más tarde, el diagnóstico era uno solo: se había progresado bastante en mejorar la exactitud y neutralidad de las especificaciones técnicas de las licitaciones públicas, así como la transparencia de los procesos de compras, pero aún había mucho espacio para avanzar en materia de gestión y preparación de procesos de compras públicas de innovación (Bason y otros, 20I3: 2I). Todas estas preocupaciones fueron reconocidas a nivel normativo mediante la dictación de la Directiva 20I4/24/UE del Parlamento Europeo y del Consejo, de 26 de febrero de 20I4, sobre Contratación Pública. Esta Directiva introdujo instrumentos específicos para la realización de procesos de compras públicas de innovación, bajo el entendido que «la adquisición de bienes, obras y servicios innovadores desempeña un papel clave en la mejora de la eficiencia y la calidad de los servicios públicos» (considerando 47).

Resulta interesante comparar el avance europeo en esta materia, con 
el desarrollo de la misma en Chile. A comienzos de siglo, cuando en Europa ya se abogaba por una mayor preocupación política y normativa por la CPTI, en Chile ni siquiera existía una regulación especialmente referida a compras públicas tradicionales. Recién en 2004 se promulgó la Ley de Compras Públicas. Curiosamente, la preocupación por las nuevas tecnologías estuvo muy presente en el diseño de esta nueva regulación, pero no se pensó en su importancia para el diseño e implementación de otras políticas públicas. Así, por una parte, se estableció un nuevo sistema de compras públicas, absolutamente electrónico y, por otra, sólo se regularon procedimientos estandarizados para el desarrollo de los procesos de compras del sector público (convenio marco, licitación pública, licitación privada y trato directo), sin tomar precauciones especiales para la adquisición de nuevas tecnologías.

Asimismo, más de diez años después, en Europa se destacaba el avance en materias de transparencia y requerimientos técnicos en relación con los procesos de compras públicas. Sin embargo, se seguía abogando por una mejor regulación de la gestión de la CPTI. Hoy en día, el diagnóstico para Chile es bastante similar. En el marco de la Agenda de Probidad y Transparencia en los Negocios y la Política impulsada por el actual gobierno, se dictó el Decreto I.4 IO de 20I5, del Ministerio de Hacienda, que vino a modificar el Reglamento de Compras Públicas. Esta nueva normativa introdujo medidas para mejorar la probidad y transparencia en los procedimientos de compras públicas, como el deber de abstención (artículo 6 bis), la delimitación de funciones (artículo I 2 bis) y la nueva regulación del Informe de la Comisión Evaluadora (artículo 4I bis).

Como se ve, tal como se denunció para el caso europeo, en Chile se ha avanzado en materia de transparencia y neutralidad, pero sigue existiendo una patente falta de consideración por la compra de innovación en el sector público. Más aun, algunas de estas reglas en favor de la transparencia en los procesos de compras públicas atentan directamente contra el éxito de los procesos de adquisición de nuevas tecnologías. Así, por ejemplo, el nuevo artículo I 2 del Reglamento de Compras Públicas establece que el órgano comprador debe procurar que participen personas distintas en las diversas etapas del proceso de compras. La estimación del gasto, la elaboración de los requerimientos técnicos y administrativos de la compra, la evaluación y la adjudicación, y la gestión del contrato deben ser realizados por funcionarios o 
equipos de trabajo distintos. En la práctica, cumplir con esta obligación es casi imposible en cualquier tipo de contratación, ya que se requeriría contar con personal con las competencias técnicas necesarias para desarrollar independientemente cada una de estas etapas. En materia tecnológica, esto cobra especial importancia, porque se requeriría que cada servicio público cuente con tantas personas y equipos expertos en nuevas tecnologías como etapas tenga el proceso de compras, cuestión que, si acaso fuese posible, redundaría en un uso altamente ineficiente de recursos públicos. A mayor abundamiento, resulta dudoso que sea preferible que el evaluador sea una persona o equipo distinto a aquel que elaboró los requerimientos técnicos. Esto implica desconocer que quien elaboró estos requerimientos suele ser quien más conciencia tiene de la necesidad del servicio y que, además, en la práctica será quien hará uso inmediato del mismo y, por ende, gestionará el contrato en los hechos. Como ejemplo, en España se ha recomendado expresamente que grupos técnicos multidisciplinarios apoyen todo el proceso de contratación de innovación, desde la etapa de preparación del procedimiento (Bernal y otros, 201 5: 27).

Con todo, debe reconocerse que han existido ciertos esfuerzos por parte de Chilecompra por avanzar en esta materia. Las Directivas 8 de 2008, y 24 de 20I 5 , mencionadas precedentemente, han entregado ciertos lineamientos a los servicios públicos para que éstos puedan realizar compras públicas relacionadas con tecnologías digitales dentro del marco de los procedimientos establecidos en la Ley de Compras Públicas. Estas directivas se limitan, en estricto seguimiento del principio de legalidad, a entregar ciertas pautas a las entidades compradoras para que procedan a realizar compras tecnológicas, como la promoción de la realización de consultas al mercado, el establecimiento de cláusulas contractuales en materia de propiedad industrial, la publicación del presupuesto estimativo y la fijación de niveles de servicio, entre otras recomendaciones. Sin embargo, ambos documentos hacen caso omiso a los requerimientos propios de la innovación pública, lo que resulta especialmente preocupante a la luz de las recomendaciones realizadas por la OCDE en materia de gobierno digital.

En primer lugar, en ambos documentos Chilecompra recomienda a todos los compradores del Estado definir con absoluta claridad la necesidad que pretenden satisfacer y, además, establecer claramente cuál es 
el servicio requerido. Sin embargo, el sector público podría promover de manera mucho más eficiente la innovación si se limitara a definir detalladamente el problema que pretende resolver, sin definir específicamente cuál es la mejor forma de hacerlo. Eso es, en esencia, la CPTI. De este modo, se aprovecharían de mucho mejor forma las capacidades del sector privado para crear una solución innovadora y eficiente, traspasando los costos de realizar un estudio acabado del mercado al lado de la oferta. Paradójicamente, ambas directivas reconocen que es un riesgo inherente a la adquisición de tecnología que los requerimientos de compra estén definidos incorrectamente, sin perjuicio de lo cual recomiendan detallar al máximo los referidos requerimientos, aumentando así las probabilidades de error y, a la vez, impidiendo a los potenciales proveedores ofertar soluciones innovadoras, en manifiesta contradicción con los compromisos contenidos en la Agenda Digital 2020. Como se mostró antes, esta Agenda contiene medidas supuestamente destinadas a promover la adquisición de soluciones innovadoras y a licitar desde la problemática y no desde la solución (medidas 5 y 38).

Sin embargo, este problema tiene su origen en la rigidez del marco jurídico nacional y, por ende, no es enteramente responsabilidad de Chilecompra. En efecto, el artículo 22 del Reglamento de Compras Públicas establece expresamente que las bases (de una licitación pública) deben contener, en lenguaje preciso y directo, las especificaciones de los bienes y/o servicios que se quieran contratar, las cuales deberán ser genéricas, sin hacer referencia a marcas específicas. La cuestión está en definir si basta o no para cumplir con este requerimiento reglamentario que se enuncien las funcionalidades que un determinado bien o servicio debe cumplir, sin expresar sus características específicas, las que pueden quedar entregadas al proveedor. Si bien, en abstracto, esto puede sonar disruptivo, al revisar contrataciones más sencillas, lo propuesto parece mucho menos disfuncional. Así, por ejemplo, al contratar una consultoría, es muy común que se establezcan los objetivos técnicos del estudio, pero que el modo de cumplir con dicho objetivo, esto es, la metodología, pueda ser libremente establecido por el proveedor en su oferta técnica.

En segundo lugar, parece inconcebible que ninguna de estas directivas haga una mención a los servicios de investigación y desarrollo $(\mathrm{I}+\mathrm{D})$, ni mucho menos a los servicios de investigación, desarrollo e innova- 
ción $(\mathrm{I}+\mathrm{D}+\mathrm{i})$, elementos fundamentales del desarrollo tecnológico en la

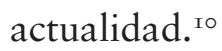

\section{COMPRA PÚBLICA DE INVESTIGACIÓN Y DESARROLLO (I+D)}

En el año 2013, Chile destinó apenas un 0,39\% del PIB a I+D, el más bajo de todos los países pertenecientes a la OCDE (OCDE, 20I 5: 97). En este contexto, no extraña que Chilecompra no haya emitido ninguna pauta para proceder a la contratación de esta clase de servicios. Por el contrario, como se revisó en el apartado precedente, en materia de compras tecnológicas, este servicio prefirió adoptar un criterio conservador, cerrándole las puertas a la innovación.

En este punto, conviene tener clara la delgada línea que separa a la CPTI de la compra directa de servicios de I+D. La CPTI supone la contratación del desarrollo de una nueva tecnología, la que es adquirida por el comprador público, sea como propietario absoluto, licenciante o bajo otra fórmula de propiedad industrial. Por su parte, la contratación de I+D no implica necesariamente la adquisición del servicio desarrollado, sino que simplemente la remuneración de las actividades de investigación y desarrollo. Si producto del trabajo de I+D se genera un servicio que interesa al ente público, éste puede decidir contratarlo o no, debiendo proceder a un nuevo proceso de compras públicas. Así, por ejemplo, si en virtud de estos servicios se genera un producto que es debidamente patentado por el proveedor, se podría proceder a contratar con este mediante trato directo, de conformidad con lo dispuesto en el artículo ıо, numeral 7, literal e) del Reglamento de Compras Públicas.

En el derecho extranjero, generalmente se identifica la contratación de servicios de I+D con la Compra Pública Precomercial. En esta clase de contratación, los riesgos propios del negocio son compartidos entre el proveedor y el órgano público contratante y no representan un subsidio o ayuda estatal (Bernal y otros, 201 5: 30). En muchos casos comprende, además, arreglos especiales en materia de propiedad intelectual.

Io. I+D+i generalmente se presenta como la evolución del concepto de I+D. Con todo, determinar si esto es así o no excede los propósitos del presente trabajo, por lo que simplemente se hará referencia al concepto de I+D. 
En este sentido, la Comunicación de la Comisión de las Comunidades Europeas, de 2007 , sobre la contratación precomercial ha identificado cinco fases para su ejecución (Apostol, 20I 2: 206). En primer lugar, el sector público debe hacer pública su intención de explorar soluciones que requieren $\mathrm{I}+\mathrm{D}$ y realizar una convocatoria abierta para recibir propuestas. En segundo lugar, los proveedores deben presentar soluciones potenciales al problema planteado, para que el contratante evalúe el diseño propuesto y seleccione a los proveedores que pasarán a la siguiente fase. En tercer lugar, los competidores deben diseñar un prototipo, el que luego es sometido a un nuevo proceso de evaluación y selección. Los prototipos seleccionados por el contratante pasan a una cuarta etapa, en la cual se desarrolla un volumen limitado de productos o servicios, a modo de serie de prueba. Se evidencia entonces un proceso piramidal, de abajo hacia arriba, en el cual los participantes de cada etapa son remunerados por los servicios prestados, sin perjuicio de quedar finalmente descalificados. Terminado el período de testeo de productos y servicios, se da por finalizado el proceso de compra precomercial. Esto quiere decir que la adquisición de los productos exitosos debe realizarse, si así se considera oportuno, recurriendo a un nuevo proceso de contratación, tal como se anunció más arriba.

En el caso de la Unión Europea, esta clase de contratación ha quedado excluida de la Directiva sobre Contratación Pública a la que se ha hecho referencia anteriormente en este trabajo. De la misma forma, la Ley Española de Contratos del Sector Público excluye de su ámbito de aplicación a

los contratos de investigación y desarrollo remunerados íntegramente por el órgano de contratación, siempre que éste comparta con las empresas adjudicatarias los riesgos y los beneficios de la investigación científica y técnica necesaria para desarrollar soluciones innovadoras que superen las disponibles en el mercado (artículo 4).

La disposición final quinta de esta misma ley regula especialmente el fomento de la contratación precomercial. En su virtud, en el presupuesto de cada Departamento ministerial y de cada organismo público de la Administración General del Estado se deben fijar las cuantías que deben necesariamente destinarse a los contratos de investigación y desarrollo que cumplan con las características recién transcritas. Aun más, una parte de 
estos presupuestos puede ser reservado especialmente para contratar a pequeñas y medianas empresas innovadoras.

En Chile, a diferencia de lo sucedido en Europa, no existe una regulación especial para la contratación de servicios de I+D, por lo que necesariamente éstos quedan bajo el alero de la Ley de Compras Públicas. En este sentido, no parece haber ningún inconveniente para que el Estado pueda solicitar la adquisición de estos servicios, los que incluso han sido definidos por el legislador. En efecto, el artículo I de la Ley que Establece un Incentivo Tributario a la Inversión en Investigación y Desarrollo, define investigación como «la búsqueda metódica que tenga por objeto generar nuevos conocimientos en el ámbito científico o tecnológico» y desarrollo como el conjunto de trabajos sistemáticos que aprovechan los conocimientos existentes obtenidos de la investigación y/o la experiencia, y está dirigido a la producción de nuevos materiales, productos o dispositivos, a la puesta en marcha de nuevos procesos, sistemas y servicios, o a la mejora sustancial de los ya existentes.

Sin perjuicio de lo anterior, la normativa nacional en materia de compras públicas no tiene la flexibilidad necesaria para ejecutar la contratación de servicios de I+D en las etapas propuestas por la Unión Europea. Dado el impulso que se ha dado a esta clase de servicios en el sector privado, Chile parece estar en un momento ideal para establecer una regulación especial para la contratación de estos servicios en el sector público.

\section{PRINCIPIO DE EFICIENCIA E I+D}

El impulso de la contratación de servicios de I+D por parte del Estado tiene directa relación con la comprensión que se tenga del principio de eficiencia, consagrado en el artículo 4 de la Ley Orgánica Constitucional de Bases Generales de la Administración del Estado, según el cual las autoridades y funcionarios públicos deben velar por la eficiente e idónea administración de los medios públicos y por el debido cumplimiento de la función pública. En este sentido, se ha postulado que en el afán de ser lo más eficientes posible, los órganos públicos muestran una tendencia a evitar la implementación de soluciones innovadoras, en la medida en que éstas son más riesgosas y, por ende, pueden ser objeto de mayores críticas en términos de accountability (Potts, 2009: 36). Así, se cierra 
la puerta a que determinados gastos propios de la innovación, como aquellos que se realizan en experimentación y búsqueda de nuevas soluciones, sean considerados eficientes. Esto sucede porque el principio de eficiencia tradicionalmente se ha entendido como

la optimización de los medios con los fines, lo que se logra seleccionando de entre varias alternativas conducentes al mismo cumplimiento, las que impliquen un menor gasto, y dentro de éstas, la que lleve al mejor cumplimiento de los objetivos de la Administración (Gardais, 2002: 328).

La definición recién anotada asume implícitamente que sólo existe una forma de alcanzar de la manera más eficiente un determinado objetivo, que esa forma es conocida o puede ser conocida y que esa solución puede ser razonablemente implementada, excluyendo así cualquier posibilidad de innovación (Potts, 2009: 35-36). No se contempla la posibilidad de que el Estado decida contratar servicios para diseñar soluciones a problemas nuevos, para los cuales no existe una oferta conocida. Cualquier iniciativa en este sentido corre el riesgo de ser considerada ineficiente, en la medida en que no logre identificar una fórmula efectiva para resolver el problema planteado y, por ende, se produzca un supuesto mal uso de recursos públicos.

Esta aproximación al concepto de eficiencia es evidentemente sesgada y atomizada y, por ende, debe ser superada. La optimización de medios y fines bien puede implicar la ejecución de diversas actividades experimentales, ensayos y errores, los que desde una perspectiva estratégica más amplia pueden ser absolutamente necesarios para proveer en el futuro servicios públicos más eficientes. En este sentido, debe distinguirse claramente entre gasto bueno (good waste) y gasto malo (bad waste) (Potts, 2009: 38). El buen gasto comprende los costos propios de la experimentación necesaria para que el Estado mantenga un constante proceso de innovación, mientras que el mal gasto comprende los costos propios de la ineficiencia. El gasto bueno es necesario; el gasto malo no. 


\section{PROCEDIMIENTOS ESPECIALES PARA LA ADQUISICIÓN DE SOLUCIONES INNOVADORAS}

\section{EL GIRO HACIA LA INTERACCIÓN CON EL PROVEEDOR}

En Europa existe una marcada tendencia a regular más y a generar nuevos procedimientos que supongan una interacción entre la entidad compradora y los potenciales proveedores de un bien o servicio. Existen diversas razones para abogar en favor de esta inclinación normativa. Para algunos (Yukins, 20I 5: 2-3), esto es necesario para evitar que los proveedores que ofrecen servicios más caros, de mejor estándar y desarrollados en función de las últimas tecnologías, tengan una posición desfavorable frente a otros competidores más económicos. Bajo esta perspectiva, se sostiene que, cuando las especificaciones técnicas de un determinado producto o servicio se encuentran predefinidas por la entidad compradora, como sucede, por ejemplo, en una licitación pública tradicional, el precio se transforma en un elemento central del proceso de adjudicación, permitiendo que sean adjudicados proveedores menos competentes, por el simple hecho de ser más económicos. Desde una perspectiva más sencilla, se ha sostenido que la interacción con el proveedor representa una herramienta que dota a los órganos públicos de mayor flexibilidad en su lucha por alcanzar de modo más eficiente sus objetivos (Moreno, 201 5: 42).

En este sentido, la Directiva 20I4/24/EU sobre Contratación Pública ha considerado «realmente necesario que los poderes adjudicadores gocen de flexibilidad a la hora de elegir un procedimiento de contratación pública que prevea negociaciones» (considerando 42), dándole especial importancia a dos procedimientos: los diálogos competitivos y la licitación con negociación. Asimismo, según esta misma Directiva, debe existir, además, un procedimiento especial para realizar contrataciones «cuando las soluciones ya disponibles en el mercado no puedan satisfacer una necesidad». En esta línea, la normativa europea incorporó en 20I4 un nuevo instrumento jurídico: la asociación para la innovación.

A continuación, se estudian estos tres procedimientos, ya que representan una novedad respecto de los procedimientos de compra más comunes, como aquellos regulados en la legislación chilena. Con todo, debe tenerse presente que el hecho de que no existan herramientas espe- 
cialmente diseñadas para adquirir innovación, no implica que las herramientas actualmente existentes no puedan ser utilizadas con esta intención. De hecho, tal como se dijo antes en este artículo, no parecen haber mayores obstáculos para que los órganos públicos comiencen a licitar desde el problema y no de desde la solución, tal como se ha propuesto en el marco de la Agenda Digital 2020. A mayor abundamiento, algunos procedimientos especiales, como los diálogos competitivos y la licitación con negociación, no tienen como único fin apoyar la contratación de nuevas tecnologías, sino que también pueden utilizarse bajo otros supuestos, como la existencia de riesgos inherentes a la contratación o, incluso, el fracaso previo de un procedimiento más tradicional.

\section{LA ASOCIACIÓN PARA LA INNOVACIÓN Y LA COMPRA PÚBLICA PRECOMERCIAL}

Como se estableció precedentemente, la asociación para la innovación es un proceso especial que, según la normativa europea, tiene por objeto «el desarrollo de productos, servicios u obras innovadores y la compra ulterior de los suministros, servicios u obras resultantes» (artículo $3 \mathrm{I}$ ). En palabras simples, se trata de la contratación de servicios de $\mathrm{I}+\mathrm{D}$ por parte del Estado. Sin embargo, no es una contratación como cualquier otra, sino una bastante especial, que se desarrolla en diversas etapas.

En primer lugar, el órgano demandante debe realizar un llamado público en el que detalla la existencia de una necesidad que no puede ser satisfecha mediante la adquisición de productos o servicios ya disponibles en el mercado. Luego, entre quienes hayan manifestado su intención de ser parte de la asociación, la autoridad contratante debe seleccionar a uno o más proveedores para que participen de ésta. Una vez realizada esta selección, comienzan propiamente las actividades de I+D y, al igual que en la compra pública precomercial, la institución demandante puede ir reduciendo el número de socios en cada una de estas fases, las que, por supuesto, han de ser debidamente renumeradas. Así, entonces, todos aquellos participantes que sean descartados en el curso de la asociación representarán, con gran probabilidad, un gasto propio de la experimentación, esto es, implicarán un gasto bueno, en los términos tratados en la sección anterior.

Sin perjuicio de lo anterior, la asociación para la innovación y la compra pública precomercial tienen diferencias importantes (Bernal y otros, 
20I 5: 4I-42). La asociación para la innovación comprende tanto las etapas propias de los servicios de I+D como la fabricación de los productos y/o la prestación de los servicios finales. En cambio, como se anotó en su oportunidad, la compra precomercial se agota con la sola prestación de los servicios de I+D y, por ende, una vez diseñado el producto o servicio respectivo, debe procederse a un nuevo proceso de contratación para la adquisición del mismo. En este nuevo proceso de compra pueden participar proveedores que no fueron parte del procedimiento precomercial. En la asociación para la innovación, por el contrario, la ejecución de los servicios o la producción de los bienes finales sólo pueden ser realizadas por un proveedor que participó de la alianza en cuestión, sea porque fue el único socio seleccionado o porque resultó adjudicado luego de haber competido en diversas etapas con otros socios seleccionados inicialmente.

Como ya se anunció, la asociación para la innovación es una institución nueva. Recién fue incluida en la normativa comunitaria europea en el año 20I 4 y, por consiguiente, aún no pueden construirse conclusiones sólidas sobre su impacto efectivo en los sistemas de innovación europeos. Así, entonces, la prudencia obliga a no emitir opiniones tajantes sobre las bondades de su importación al sistema jurídico chileno. Hecha esta prevención, resulta importante hacer presente que en Chile existe un modelo de gestión de proyectos que, aun sin un reconocimiento legal explícito, se sitúa en un punto intermedio entre la asociación para la innovación y la compra pública precomercial.

En efecto, como se anotó precedentemente, una de las líneas de trabajo del Laboratorio de Gobierno consiste en la gestión de proyectos de innovación en el sector público. Para el desarrollo de estos proyectos se ha elaborado un modelo que consta de seis etapas: exploración institucional, descubrimiento del desafío, generación de ideas, prototipado y testeo, pilotaje y evaluación, y, finalmente, gestión del conocimiento.

En relación con la compra pública precomercial, este modelo de innovación supone un avance en la medida que aquélla finaliza, por regla general, con la elaboración de un número limitado de productos a modo de serie de prueba. En cambio, en el marco de la iniciativa impulsada por el Laboratorio de Gobierno, se desarrolla una etapa de pilotaje y evaluación, que permite luego la elaboración de recomendaciones concretas para la replicabilidad o ejecución de la solución innovadora respectiva 
(gestión del conocimiento). Con todo, al igual que en el caso de la contratación precomercial, el modelo en cuestión no supone la adquisición definitiva del bien o servicio final, a diferencia de lo sucedido en el caso de la asociación para la innovación.

Ahora bien, aun cuando se trata de un modelo sencillo y que, además, no cuenta con la solidez formal de un procedimiento regulado, representa una oportunidad ideal para realizar gastos legítimos en experimentación (gasto bueno). Teniendo presente que muchos servicios públicos cuentan con presupuestos acotados, el Laboratorio de Gobierno podría transformarse en una especie de centro I+D del Estado, al cual las instituciones públicas recurran con una necesidad para la cual no existe una solución en el mercado (o para la cual podría existir otra más eficiente), para que apoye técnica y financieramente las actividades de I+D requeridas para generar la referida solución. En este sentido, podrían introducirse al modelo elementos competitivos entre proveedores $y$, por supuesto, una nueva etapa, en la cual el organismo demandante adquiera los bienes o servicios finales. Así, se podría avanzar desde un modelo bien construido, pero no formalizado, a un procedimiento regulado, pero flexible, al que las instituciones puedan recurrir para realizar gastos en experimentación, de la manera más eficiente posible.

\section{LA LICITACIÓN CON NEGOCIACIÓN Y LOS DIÁLOGOS COMPETITIVOS}

La normativa europea establece, además, otros dos procedimientos en que existe una fuerte interacción entre los proveedores potenciales de un servicio y la autoridad contratante: los diálogos competitivos y la licitación con negociación. Ambos procedimientos son muy similares y, de hecho, según lo establecido en la mencionada Directiva europea, son aplicables al concurrir exactamente las mismas causales, entre las cuales se encuentra la contratación de obras, suministros o servicios que incluyan un proyecto o soluciones innovadoras (artículo 26).

El procedimiento con diálogo competitivo tiene por objeto determinar y definir los medios más idóneos para la satisfacción de una necesidad, mediante un debate en el cual se discuten todos los aspectos de una determinada contratación (artículo 30). En otras palabras, los diálogos competitivos son utilizados por el órgano contratante cuando éste no tiene claridad sobre la forma más adecuada de solucionar un determi- 
nado problema (Telles y Butler, 20I4: I I). Este procedimiento se inicia con un llamado público. Luego, se procede a la selección de uno o más competidores para que participen del proceso de diálogo, mediante el cual se trata de definir una determinada solución. Finalmente, una vez precisados los requerimientos de la solución que se debe contratar, la autoridad demandante debe solicitar a los participantes de los diálogos que hagan llegar sus ofertar para desarrollar la referida solución y así proceder a adjudicar el bien o servicio respectivo.

En Chile, la institución más similar a este procedimiento es la consulta al mercado, o RFI, como se conoce típicamente, en razón de su nombre en inglés (request for information). De acuerdo al artículo I 3 bis de del Reglamento de Compras Públicas, este mecanismo puede consistir en un proceso formal de consultas o en reuniones con proveedores, que se realizan mediante llamados públicos y abiertos, con el objeto de obtener cualquier información que el órgano comprador requiera para la confección de unas bases de licitación. En semejanza con las hipótesis de procedencia de los diálogos competitivos en Europa, Chilecompra ha recomendado, a través de su Directiva 24, recurrir a una RFI cuando la entidad compradora ignore los aspectos técnicos necesarios y suficientes para definir un requerimiento de compra en materia tecnológica. Con todo, existen menos incentivos para apoyar al órgano público, en comparación con los diálogos competitivos, porque en la consulta al mercado aún no ha comenzado el procedimiento competitivo, mientras que en este caso los proveedores dialogan habiendo sorteado una primera etapa de selección y, por ende, tienen mayores expectativas de adjudicarse la provisión del bien o servicio respectivo.

El procedimiento de licitación con negociación, por su parte, es bastante similar. Sin embargo, en este caso la entidad compradora tiene claridad sobre el objeto de la contratación y, de hecho, el pliego respectivo, según la Directiva europea, debe contener las características exigidas para los suministros, obras o servicios que se pretenda contratar (artículo 29). Además, este procedimiento se distingue de los diálogos competitivos en la medida en que los proveedores inicialmente seleccionados son invitados a presentar una oferta inicial, la que sirve de base para las negociaciones posteriores. Cuando éstas llegan a su fin, la institución demandante invita a los participantes a presentar una nueva oferta, la que tiene el carácter de definitiva. Sin embargo, de la misma forma que 
en la asociación para la innovación y en el diálogo competitivo, el órgano contratante se encuentra habilitado para dividir el procedimiento en fases sucesivas, con la intención de ir reduciendo progresivamente el número de participantes.

Como ya es evidente a estas alturas, los tres procedimientos especiales regulados en la Directiva Europea sobre Contratación Pública y que son objeto de la presente sección comparten características muy similares. Frente a esta situación, existen diversas opiniones (Telles y Butler, 20I4: IO-II). Para algunos, la existencia de diversos procedimientos supone un mayor poder de elección para los órganos contratantes. Para otros, esto sólo contribuye a generar confusión y, en este sentido, puede provocar que las autoridades nacionales opten por utilizar los procedimientos que les son más familiares, dejando a los demás en desuso.

\section{CONCLUSIÓN}

Sin duda, la gran conclusión de este trabajo dice relación con la inconveniencia de utilizar enfoques atomizados al estudiar, regular o enfrentar de cualquier modo los desafíos en materia tecnológica. La falta de una visión estratégica o, a lo menos, de una visión que contemple dos o más factores, ha excluido del concepto de CPI ciertas adquisiciones que si bien pueden parecer tradicionales, responden a una estrategia más amplia en materia de innovación; que en el afán de dotar de mayor transparencia al sistema de compras públicas, se impongan trabas a la adquisición de nuevas soluciones tecnológicas; y que, con la intención de ser eficientes en el corto plazo, los órganos públicos se muestren reacios a emprender iniciativas de mediano o largo plazo en favor de la innovación.

El Estado debe entonces comprender que en la era del gobierno digital, los servicios públicos deberán recurrir al uso intensivo de las tecnologías en el diseño e implementación de políticas públicas, desde una perspectiva multidisciplinaria que permita procesos de ensayo y error en favor de la innovación (Dawes, 20I3: 53). Desde la perspectiva del diseño, la utilización de mejores soluciones para el tratamiento de datos, por ejemplo, puede resultar fundamental para asistir el proceso de toma de decisiones en el sector público. Por su parte, desde la perspectiva de la implementación, sin duda existe un desafío por incorporar más tecnologías en la prestación de los servicios que entrega el Estado. 
El discurso en favor de la innovación debe ser reemplazado por acciones concretas en este sentido. Deben incorporarse en la Administración Pública los cargos e instituciones que exige la existencia de un gobierno digital, de acuerdo a los máximos estándares internacionales señalados por la OCDE. Debe, también, actualizarse la normativa nacional en materia de compras públicas, para transformar al Estado en un verdadero consumidor de innovación. En esta línea, Chile ha realizado muchos avances por promover la innovación privada. Ha llegado la hora de la innovación pública.

\section{REFERENCIAS}

Apostol, Anca Ramona (2OI2). «Pre-commercial procurement in support of innovation: Regulatory effectiveness». Public Procurement Law Review, (6): 2I3-225. Disponible en http://eupian.eu/upload/ knowdlegdebase/20I2_PPLR_Issue \% 206_PCP\% 20in \% 20support \% 20of\% 2oinnovation_Regulatory\% 2oeffenctiveness.pdf.

Appelt, Silvia y Fernando Galindo-Rueda (20I6). "Measuring the link between public procurement and innovation». En OECD Science, Technology and Industry Working Papers (París: OECD Publishing), 7 de julio de 20I6. Disponible en: Io.I787/5jlvc7slıw7h-en.

AschHoff, Birgit y Wolfgang Sofka (2008). "Innovation on demand: Can public procurement drive market success of innovations?». ZEW Discussion Papers, o8 (052). Disponible en https://www.econstor.eu/ handle/IO4I9/24748.

Bason, Christian, Hugo Hollanders, César Hidalgo, Rainer Kattel, Gina Korella, Christine Leitner, Bernard Le Masson, Mariana Mazzucato, Alina Mungiu-Pippidi, Marga Pröhl y Jan Oravec (2013). «Powering European public sector innovation: Towards a new architecture». Report of the expert group on public sector innovation, European Comission.

Benington, John y Mark Moore (20I I). «Public value in complex and changing times». En John Benington y Mark Moore (editores), Public value: Theory and practice (pp. I-30). Reino Unido: Plagrave Macmillan.

Bermúdez, Jorge (2015). «El principio de legalidad y la nulidad de derecho público en la Constitución Política. Fundamentos para la apli- 
cación de una solución de derecho común». Revista de Derecho Público, 70: 273-285. Disponible en http://www.revistaderechopublico. uchile.cl/index.php/RDPU/article/view/37764.

Bernal, Miguel y José María Jimeno (coords.), Patricia Valcárcel, Rafael Fernández, Beatriz Requeijo y Antonio Miño (201 5). "Guía 2.0 para la compra pública de innovación». Subdirección General de Fomento de la Innovación Empresarial del Ministerio de Economía y Competitividad por miembros del Observatorio de Contratación Pública, España.

DAwEs, Sharon (2013). «Avances en gobierno electrónico: La conexión entre práctica, conocimiento e investigación». Gestión y Política Pública, 22: 49-67. Disponible en http://www.scielo.org.mx/scielo. php?script=sci_arttext\&pid=SI 405-I07920I3000400002.

De Vries, Hanna, Victor Bekkers y Lars Tummers (20I 5 ). «Innovation in the public sector: A systematic review and future research agenda». Public Administration, 94 (I): I46-I66. Disponible en http://onlinelibrary.wiley.com/doi/IO.I I I I/padm.I 2209/pdf.

Edquist, Charles y Leif Hommen (2000). «Public technology procurement and innovation theory». En Charles Edquist, Leif Hommen y Lena Tsipouri (editores), Public technology procurement and innovation. Nueva York: Springer Science \& Business Media.

Edquist, Charles y Jon Mikel Zabala-Iturriagagoitia (2OI2). «Public procurement for innovation as mission-oriented innovation policy». Research Policy, 4I (IO): I757-I 769. Disponible en http://www. sciencedirect.com/science/article/pii/So0487333 I $200220 \mathrm{X}$.

GARDAIs, Gabriela (2002). «El control de legalidad y la eficiencia y eficacia como principios jurídicos fiscalizables». Revista de Derecho (Universidad Católica de Valparaíso), 23: 323-34I. Disponible en http://www.rdpucv.cl/index.php/rderecho/article/viewArticle/5 I I.

Georghiou, Luke, Jakob Edler, Elvira Uyarra y Jillian Yeow (2OI4). «Policy instruments for public procurement of innovation: Choice, design and assessment». Technological Forecasting and Social Change, 86: I-I2. Disponible en http://www.sciencedirect.com/science/ article/pii/So0401625I3002552.

Hartley, Jean, Eva Sørensen y Jacob Torfing (2013). «Collaborative innovation: A viable alternative to market competition and organizational entrepreneurship». Public Administration Review, 73 (6): 
82 I-830. Disponible en http://onlinelibrary.wiley.com/doi/IO.I I I I/ puar.I2I36/full.

Hoppe, Eva, David Kusterer, Patrick Schmitz (20I3). «Public-private partnerships versus traditional procurement: An experimental investigation». Journal of Economic Behavior \& Organization, 89: I45I66. Disponible en http://www.sciencedirect.com/science/article/pii/ SoI67268IIIOOI2II.

Kattel, Rainer, Aleksandrs Cepilovs, Wolfgang Drechsler, Tarmo Kalvet, Veiko Lember y Piret Tõnurist (20I2). "Can we measure public sector innovation. A literature review». LIPSE Project Paper. WP 6 Social innovation indicators. Disponible en http://lipse.org/ userfiles/uploads/kattel\% 2oet \% 20al\% 2oegpa \% 2oversion.pdf.

Kelly, Gavin, Geoff Mulgan y Stephen Muers (2002). "Creating public value. An analytical framework for public service reform». Strategy Unit, Cabinet Office, UK.

Kochanova, Anna, Zahid Hasnain y Bradley Larson (20I6). «Does e-government improve government capacity? Evidence from tax administration and public procurement». World Bank, Policy Research Working Paper 765. Disponible en http://documents.worldbank. org/curated/en/33448 I468I93734893/Does-e-government-improvegovernment-capacity-evidence-from-tax-administration-and-publicprocurement.

Lember, Veiko, Rainer Kattel y Tarmo Kalvet (20I4). «Public procurement and innovation: Theory and practice». En Veiko Lember, Rainer Kattel y Tarmo Kalvet (editores), Public procurement, innovation and policy. International perspectives. Berlín, Heidelberg: Springer.

McIver, William J. y Ahmed K. Elmagarmid (editores) (2008). Advances in digital government: Technology, human factors, and policy. Nueva York: Kluwer Academic Publishers.

Moreno, Juan Antonio (20I 5 ). El nuevo derecho de la contratación pública de la Unión Europea. Directivas 4.०. Oxford: Chartridge Books Oxford.

Mulgan, Geoff (2007). «Ready or not?: Taking innovation in the public sector seriously». NESTA publications. Disponible en http://www.nesta.org.uk/publications/ ready-or-not-taking-innovation-public-sector-seriously. 
Mulgan, Geoff y David Albury (2003). «Innovation in the public sector». Londres, Strategy Unit, Cabinet Office.

Mulgan, Geoff, Simon Tucker, Rushanara Ali y Ben Sanders (2007). "Social innovation: what it is, why it matters and how it can be accelerated». Working Paper, Skoll Centre for Social Entrepreneurship, Saïd Business School, University of Oxford. Disponible en http://eureka.sbs.ox.ac.uk/76I/.

OCDE (2OI4). "Recommendation of the Council on Digital Government Strategies Adopted by the OECD Council on I 5 July 20I4".

-. (20I 5 ). «OECD Science, Technology and Industry Scoreboard 201 5 : Innovation for growth and society». OECD Publishing, París. Disponible en http://dx.doi.org/Io.I787/sti_scoreboard-20I 5-en.

-. (2016) «Digital Government in Chile: Strengthening the Institutional and Governance Framework» OECD Publishing, París. Disponible en http://dx.doi.org/IO.I787/9789264258013-en.

PotTs, Jason (2009). "The innovation deficit in public services: The curious problem of too much efficiency and not enough waste and failure». Innovation: Management, Policy \& Practice, II (I): 3443. Disponible en http://www.tandfonline.com/doi/abs/I0.5172/ impp.453.II.I.34.

RedDICK, Christopher (20I2). Public Administration and Information Technology. Burlington, MA: Jones \& Bartlett Learning.

Rivero, Ricardo y Amador Fernández (2016). «La Administración sin papel: registro, expediente, archivo electrónico ¿estamos preparados?». Revista Vasca de Administración Pública. Herri-Arduralaritzako Euskal Aldizkaria, I05: 453-471. Disponible en https://dialnet. unirioja.es/servlet/articulo? codigo $=5664265$.

RodAl, Eduardo (2004). «Programa para el establecimiento del gobierno electrónico en América Latina y el Caribe: conceptos, estrategias y aplicaciones que el Banco Interamericano de Desarrollo viene desarrollando en la región». Revista de Administración Pública, I Iо: 337-348. Disponible en https://revistas-colaboracion.juridicas.unam. mx/index.php/rev-administracion-publica/article/view/I $9253 / 17345$.

Rolfstam, M. (2OI2). «Understanding public procurement of innovation: definitions, innovation types and interaction modes. Innovation Types and Interaction Modes». Social Science Research Network. Disponible en https://ssrn.com/abstract=20I 488. 
SÁNCHEZ, Carmina y Marcelo LASAGNa (2013). Innovación pública: Un modelo de aportación de valor. Santiago: RIL Editores.

Telles, Pedro y Luke Butler (20I4). «Public Procurement Award Procedures in Directive 20I4/24/EU». En Francois Lichere, Roberto Caranta y Steen Treumer (editores), Novelties in the 2014 Directive on Public Procurement. Djof Publishing. Disponible en https://ssrn.com/ abstract $=2443438$.

Yukins, Christopher (20I 5 ). «Public Procurement Law: Key International Developments in 2014. Part I: An American Perspective on the New European Public Procurement Directives». West Government Contracts Year en Review Conference Covering 20I4 Conference Briefs, Thomson Reuters.

\section{SOBRE EL AUTOR}

César Abusleme Mardones es abogado. Licenciado en Ciencias Jurídicas y Sociales por la Universidad de Chile. Se desempeña como Director Jurídico del Programa de Modernización del Sector Público, Ministerio de Hacienda, Chile. Su correo electrónico es cesar.abusleme@ hacienda.gov.cl.

Artículo recibido el I I de noviembre y aceptado para su publicación el I de diciembre de 2016. 